\section{Temporospatial distribution of Culicoides species and Culicoides imicola in northern Jordan}

\author{
Rami M. Mukbel, 1 \\ Abd Almajeed M. Alajlouni,1 \\ Musa A. Alshehabat,2 \\ Siham M. Bataineh, ${ }^{3}$ \\ Ahmad M. Al-Majali2 \\ 1Department of Veterinary Basic \\ Sciences; ${ }^{2}$ Department of Veterinary \\ Clinical Sciences, Faculty of Veterinary \\ Medicine; ${ }^{3}$ Department of Civil \\ Engineering, Faculty of Engineering, \\ Jordan University of Science and \\ Technology, Irbid, Jordan
}

\section{Abstract}

The aim of this study was to estimate geographical distribution of Culicoides species and Culicoides imicola in northern governorates of Jordan. The study was conducted by placing light traps in four climatically different geographical locations during 2011. Suitability maps were created by layering and compiling climatic parameters into the GIS data to highlight locations and time suitable for growth of C. imicola. Collected insect samples were assorted by morphology to identify Culicoides species. Molecular analysis was used to identify Culicoides spp. and $C$. imicola. In total, 25,196 insects were trapped of which 3491 (12.7\%) were morphologically identified as Culicoides spp. The highest counts Culicoides spp. were recorded in Deir Alla (47\%), Banikenaneh (31\%) and Al-Shouneh (21\%) respectively. The peak activity was recorded during August through October. Morphological identification failed to identify Culicoides species in 4 locations while polymerase chain reaction analysis identified Culicoides spp. in all locations except Al-mafraq. $C$. imicola could only be identified in Deir Alla, Bani-kenaneh and Al-Shouneh. There was no evidence of viral genome of epizootic hemorrhagic disease virus, blue tongue virus and bovine ephemeral fever virus in the trapped midges.

\section{Introduction}

Culicoides are small biting midges belonging to the Ceratopogonidae family, which are of human and veterinary significance. ${ }^{1}$ There are more than 1300 identified Culicoides species worldwide. Taxonomic identification of
Culicoides species is traditionally based on morphological characterization of the insect's wing pattern. ${ }^{2}$ Culicoides spp. are characterized by the presence of two radial cells that are absent from other genera in the family. ${ }^{3}$ Morphological identification of Culicoides spp. might be time-consuming and difficult. ${ }^{4}$

Culicoides species have been reported to be a vector of many economically significant diseases in horses and ruminant including African horse sickness and blue tongue. 5 The African horse sickness is considered a notable disease by the World Organization for Animal Health. In the Middle East, Culicoides imicola has been implicated to play a role in the occurrence of two economically significant diseases that affected the cattle industry including epizootic hemorrhagic disease (EHD) and bovine ephemeral fever. ${ }^{6}$ In 2006, serotypes 6 and 7 of EHD virus were detected in Jordan Valley in Israel. ${ }^{7}$ In Jordan, few suspected cases have been reported in the same year to be serologically positive for the EHD virus however the virus has never been isolated. ${ }^{8}$ Furthermore, serotype 7 of EHD virus was detected in Turkey in 2007. ${ }^{9}$ Till today, there has been no evidence for the described viruses, although Culicoides have been recorded in the region. Since then, no reported research efforts were undertaken to further investigate and characterize occurrence of Culicoides spp. in Jordan. Therefore, the broad goals of the research presented here were: to evaluate the spatiotemporal distribution of Culicoides spp. and $C$. imicola in northern governorates of Jordan and to establish vector suitability map to help highlight locations and time suitable for occurrence of $C$. imicola. Furthermore, to test the presence of epizootic hemorrhagic disease virus, blue tongue virus and bovine ephemeral fever virus in the morphologically identified pooled samples by real time polymerase chain reaction (RT-PCR). Our hypothesis is that $C$. imicola does occur in Jordan and will be identified in some environmentally suitable locations.

\section{Materials and Methods}

\section{Collection sites}

Insect samples were collected from eight geographical sites located within five governorates in northern Jordan during June through November 2011. Those locations are: Irbid (Irbid city, Bani-Kenaneh, Elmazar and Al-Shouneh); Al-mafraq (Al-mafraq city); Jarash (Jarash city); Zarqa (Ad-Dulayl) and Albalqa (Deir Alla). Study locations were classified based on climate into: Deir Alla and AlShouneh where the climate is characterized as warm steppe climate; Ad-Dulaul and Al-mafraq have a cool desert climate; Jerash, Irbid and
Correspondence: Rami M. Mukbel, Department of Veterinary Basic Sciences, Faculty of Veterinary Medicine, Jordan University of Science and Technology, P.0. Box 3030, Irbid 22110, Jordan. Tel: +962.795170357 . Fax: +962.27201081 .

E-mail: rmmukbel@just.edu.jo

Key words: Biting midges; climate; GIS; vector; virus.

Acknowledgements: the work was supported by a grant from USAID MERC program (M29-060).

Conflict of interest: the authors declare no potential conflict of interest.

Contributions: RMM, AMAA, AMA, research design; RMM, AMAA, conducting the research; RMM, AMAA, MAA, data analysis and writing manuscript; SMB, GIS analysis, AMA manuscript reviewing and obtaining funding.

Received for publication: 5 October 2015. Accepted for publication: 27 October 2015.

This work is licensed under a Creative Commons Attribution NonCommercial 4.0 License (CC BYNC 4.0).

(C) Copyright R.M. Mukbel et al., 2016

Licensee PAGEPress srl, Italy

Veterinary Science Development 2016; 6:6231

doi:10.4081/vsd.2016.6231

Bani-Kenaneh have a warm temperate climate and Elmazar which considered as cool temperate rainy climate. 10

\section{Collection of insects}

Insect samples were collected from farms were permitted by owner after signing a consent form. Insects were collected using CDClight traps. The traps were placed inside and around the cattle farms one meter above ground level in the dark. 3-4 traps were placed overnight per each farm and the trapped insects were then transferred to the laboratory in a container containing sugar solutionsoaked cotton.

\section{Processing and analysis}

Upon arrival to the parasitology laboratory, the collected insect samples were washed with mild soap detergent. Initial morphological identification of the Culicoides spp. was based on the wing pattern using dissecting and light microscope as described by Glukhova. ${ }^{11}$ The morphologically identified Culicoides spp. were assorted into groups of 10-100 insects and stored in a $2 \mathrm{~mL}$ microcentrifuge tube containing $0.5 \mathrm{~mL} \mathrm{PBS}$ at $-80^{\circ} \mathrm{C}$ for further molecular analysis. The identified samples were homogenized using Omni Bead Ruptor 
Homogenizer (Omni International, GA, USA). The genomic DNA was isolated from insects' samples using Promega Wizard® Genomic DNA Purification Kit according to manufacturer instructions (Promega, WI, USA). Oligonucleotide specific-Culicoides spp. and $C$. imicola primers for the PCR assay were selected based on the published nucleotide sequence to amplify DNA fragment of the ITS-1 rDNA subunit in the Culicoides spp. genome and for amplification of 316 bp DNA fragment of the ITS- 1 rDNA subunit in the $C$. imicola genome as described by Cetre-Sossa and colleagues. ${ }^{12}$

The morphologically identified insect pooled samples were tested for presence of epizootic hemorrhagic disease virus, blue tongue virus and bovine ephemeral fever virus. The genome of the described viruses was extracted using extraction kits (Intron, South Korea) according to the manufacturer's instructions. The samples were tested for the presence of those viruses using specific RT-PCR kits according to the manufacturer's instructions (Primer Design, UK).

\section{Climate parameters}

Climate parameters used to establish suitability maps for study locations for the occurrence of $C$. imicola were ambient daily temperature, wind speed and humidity. The climate parameters were monthly averaged and combined to create vector suitability maps using
Arc Gis (Version 9.3) during the period June through November 2011. The reported optimal temperature, humidity and wind speed for $C$. imicola peak activity is $\geq 18 \leq 28^{\circ} \mathrm{C}, 75-85 \%$ and $\leq 2.5 \mathrm{~m} / \mathrm{s}$ respectively. $13-15$ The study locations were classified based on potential risk value ranging from low to high.

\section{Results}

The number of collected insect samples during the study period is characterized in Table 1. The total numbers of collected insects were 25,196 samples of which 3194 samples (12.7\%) were morphologically identified as a Culicoides spp. according to the wing pattern. There was a considerable variation in the occurrence of Culicoides spp. in the studied region. The lowest occurrence of Culicoides activity was in recorded in June while the highest activity was recorded in September. In the study regions, 47,31 and $21 \%$ of the trapped Culicoides were recorded in Deir Alla, Bani-Kenaneh and $\mathrm{Al}$-Shouneh respectively.

The analysis of the PCR data indicated presence of Culicoides spp. in all study locations except Al-mafraq. $C$. imicola could be identified only in three locations: Al-Shouneh, Banikenaneh and Deir Alla. Morphological characterization could not identify Culicoides spp. in

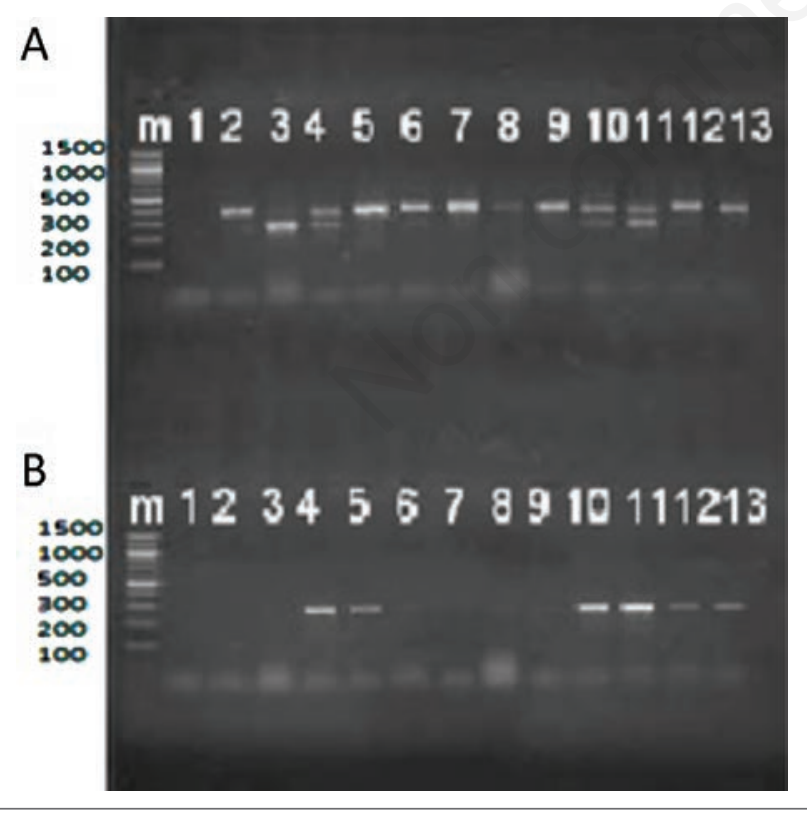

Figure 1. Representative gel electrophoresis for polymerase chain reaction products. (A) Indicate amplification of the Culicoides species common ITS1 sequence (PanCulF/PanCulR). (B) Indicates amplification of $C$. imicola ITS1 specific sequence. $M$ indicates 100 bp DNA ladder, lane 1 indicates negative control, lanes 2, 6, 7, 8, 9, 11, 12, 13 indicate samples from Deir Alla, lane 3 indicates samples from Ad-Dulayl, lane 4 indicates samples from Bani-kenaneh, lanes 5 and 10 indicates samples from Al-Shouneh. four locations while PCR findings confirmed that some of the insect samples initially presumed to be morphologically non-Culicoides are actually identified as Culicoides (Figure 1, Table 2). The RT-PCR findings suggest absence of epizootic hemorrhagic disease virus, blue tongue virus and bovine ephemeral fever virus in the tested insect samples.

The generated suitability map highlighted locations and time suitable for occurrence of C. imicola. Analysis of the suitability maps suggests that $C$. imicola does occur in particular areas in Jordan. The data of the suitability maps are color-coded into Table 2 and Figure 2. It is noticeable that regions where $C$. imicola identified have an altitude of lower than approximately $400 \mathrm{~m}$ AMSL (Table 2).

\section{Discussion}

The current study was undertaken to estimate occurrence and geographical distribution of Culicoides spp. and $C$. imicola in four climatically different regions in northern Jordan during 2011. The suitability maps identified geographical locations and time suitable for the occurrence of $C$. imicola.

The abundance rate of insects morphologically characterized as Culicoides app. was estimated to be approximately $12.7 \%$ of the total trapped insect samples. However, authors speculate that the actual abundance rate of Culicoides in the study locations might be higher. Findings of PCR analysis showed that pooled samples, initially characterized as nonCulicoides spp., actually identified as Culicoides spp. The authors concluded that some of the captured insect samples could not be easily classified morphologically. Previous

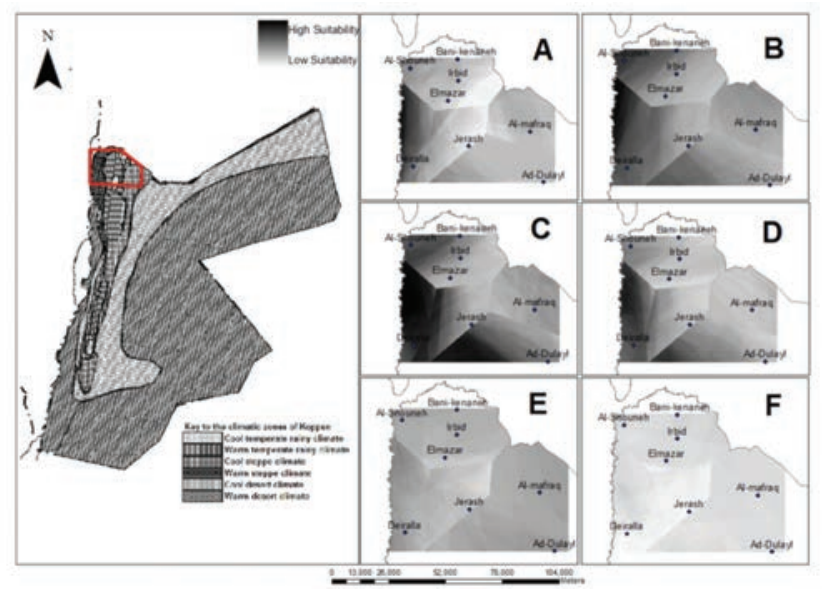

Figure 2. Categories of the climatic zones of the study locations and the monthly geographical information system suitability maps for $C$. imicola for the study regions from June (A) through November (F) during 2011. The darker the color means the more suitable conditions for occurrence of $C$. imicola. 
research efforts have suggested that identification of some Culicoides spp. based on morphological traits might be challenging. ${ }^{6,17}$ This might explain the mischaracterization of some insect samples based on morphology. The PCR analysis therefore could potentially be more practical to accurately identify mixed collections of insects especially when examining massive number of samples during outbreaks. ${ }^{18,19}$ In our study, the PCR analysis identified Culicoides spp. in all study locations except Al-mafraq area while morphological characterization could only identify Culicoides in four locations and $C$. imicola were identified in three locations. Previous research has characterized occurrence and distribution of different Culicoides spp. For instance, the prevalence rate of the identified Culicoides species varied depending on the study region. In Zimbabwe and Namibia, $C$. imicola was the most abundant Culicoides spp. with a prevalence rate of 80.4 and $99.4 \%$ respectively. ${ }^{20,21}$ In Kingdom of Saudi Arabia, abundance rate of $C$. imicola was estimated to be $19 \%$ of the morphologically identified Culicoides spp. and the peak activity was recorded during May of 20042006.22 While in Israel, the peak activity of $C$. imicola was recorded in September. ${ }^{23}$ In here, there was obvious monthly variation in the occurrence rate and monthly distribution of Culicoides spp. and $C$. imicola. Our results showed that Culicoides spp. are most abundant during September while $C$. imicola can occur during July, September, October and November. There are existing substantial research efforts that investigated the effect of several factors on the activity of Culicoides spp. including air temperature, wind speed, humidity, soil moisture, topography, land use, vegetation cover, presence of ruminant hosts. ${ }^{24,25}$ In the current work, suitability map for $C$. imicola was created by layering and compiling climate data from trapping site into the geographical information system. 14 The suitability map identified geographical regions and time that match optimal conditions suitable for occurrence of $C$. imicola. There is a considerable variation in the climatic parameters and altitude of study areas. The altitude of the study regions ranged from $223 \mathrm{~m}$ BMSL to 808 m BMSL. ${ }^{26}$ It is noticeable that Culicoides was found in all location except Al-mafraq while $C$. imicola was found in three regions. In here, the effect of the availability of the surface water on the activity of $C$. imicola was not studied. However, it is worth mentioning that areas found that have more abundant $C$. imicola had relatively more abundant surface water sources such as open irrigation systems, springs and man-made ponds. The combination of climatic parameters in addition to the availability of water source may have provided suitable environment for occurrence of $C$. imicola. It is noticeable that regions where $C$. imicola identified have an altitude of lower than approximately $400 \mathrm{~m}$ AMSL.

In here, there was no molecular evidence of the viral genome in the trapped Culicoides midges. However, at the time of the insect's collection there were no reports of clinical cases related to the described viruses in the study regions. Previous reports have suggested that detection of vector-borne viral diseases is usually low even during occurrence of outbreaks. Experimental study designs have only reported of less than $1 \%$ recovery rate of viruses from insects fed on infected blood. 27

In this work, there are only a few limitations. The study regions were limited to the northern parts of Jordan. Establishing effective preventive or control strategies require further research that covers more regions over multiple years span to overcome the climatic fluctuations and variation. The significance of study reported here is considered the first research efforts to document occurrence of $C$. imicola in Jordan. The results reported here highlighted areas suitable for optimal $C$. imicola infestation. Understanding the spatiotemporal distribution of $C$. imicola is of clinical veterinary significance when implementing diagnostic and surveillance measures particularly during occurrence of outbreaks.

Table 1. Total monthly number of trapped insects in the study regions.

\begin{tabular}{lcccccc} 
Collection area & June & July & Aug & Sep & Oct & Nov \\
Deir Alla & 0 & 0 & $752(344)$ & $2585(550)$ & $981(400)$ & $628(228)$ \\
Elmazar & $265(0)$ & $437(0)$ & $309(0)$ & $209(0)$ & $322(0)$ & NC \\
\hline Aḍ-Dulayl & $4356(0)$ & $845(0)$ & $276(0)$ & $756(0)$ & $403(0)$ & $423(0)$ \\
Al-mafraq & 0 & 0 & $635(0)$ & $817(0)$ & $400(0)$ & $185(0)$ \\
\hline Jerash & $645(0)$ & $237(0)$ & $209(0)$ & $414(0)$ & $657(0)$ & NC \\
Al-Shouneh & $756(0)$ & $894(130)$ & $840(120)$ & $851(143)$ & $1040(150)$ & $760(133)$ \\
Irbid & $345(0)$ & $834(0)$ & $548(0)$ & $430(0)$ & $212(0)$ & NC \\
Bani-kenaneh & $888(220)$ & $708(145)$ & $745(320)$ & $973(187)$ & $547(124)$ & $\mathrm{NC}$ \\
\hline
\end{tabular}

Parenthesis indicates the number of Culicoides species identified morphologically; NC, no collection was performed.

Table 2. Summary for detection of Culicoides species and C. imicola using polymerase chain reaction.

\begin{tabular}{|c|c|c|c|c|c|c|c|}
\hline Collection area & Elevation (m) & June & July & Aug & Sep & Oct & Nov \\
\hline Deir Alla & -223 & $*$ & $*$ & $\mathrm{Cu} / \mathrm{Cm}^{*}$ & $\mathrm{Cu} / \mathrm{Cm}^{*}$ & $\mathrm{Cu} / \mathrm{Cm}^{*}$ & $\mathrm{Cu} / \mathrm{Cm}$ \\
\hline Elmazar & 808 & $\mathrm{Cu}$ & & & $\mathrm{Cu}$ & & \\
\hline Aḍ-Dụlayl & 579 & $\mathrm{Cu}$ & & & $\mathrm{Cu}$ & & \\
\hline Al-mafraq & 705 & & & & & & \\
\hline Jerash & 620 & & & & $\mathrm{Cu}$ & & \\
\hline Al-Shouneh & -201 & $\mathrm{Cu}^{*}$ & $\mathrm{Cu} / \mathrm{Cm}^{*}$ & $\mathrm{Cu}^{*}$ & $\mathrm{Cu}^{*}$ & $\mathrm{Cu} / \mathrm{Cm}$ & $\mathrm{Cu}$ \\
\hline Irbid & 568 & & $\mathrm{Cu}$ & & & & \\
\hline Bani-kenaneh & 417 & $\mathrm{Cu}$ & $\mathrm{Cu} / \mathrm{Cm}^{*}$ & $\mathrm{Cu}^{*}$ & $\mathrm{Cu}$ & $\mathrm{Cu}$ & \\
\hline
\end{tabular}

$\mathrm{Cu}$, detection of Culicoides species; $\mathrm{Cm}$, detection of $C$. imicola; *high risk for occurrence of $C$. imicola according to the generated suitability maps. 


\section{References}

1. Meiswinkel R, Gomulski LM, Delecolle JC, et al. The taxonomy of Culicoides vector complexes - unfinished business. Vet Ital 2004;40:151-9.

2. Mushi EZ, Isa JF, Chabo RG, et al. Culicoides associated with dairy cows at Sebele, Gaborone, Botswana. Trop Anim Health Prod 1998;30:305-7.

3. Wirth WW, Hubert AA. The Culicoides of Southeast Asia (Diptera: Ceratopogonidae). Memoirs of the America Entomological Institute. Gainesville, USA; 1989. pp 508.

4. Nielsen SA, Kristensen M. Delineation of Culicoides species by morphology and barcode exemplified by three new species of the subgenus Culicoides (Diptera: Ceratopogonidae) from Scandinavia. Parasit Vectors 2015;8:151.

5. Guichard S, Guis H, Tran A, et al. Worldwide niche and future potential distribution of Culicoides imicola, a major vector of bluetongue and African horse sickness viruses. PLoS One 2014;9:e112491.

6. Morag N, Saroya Y, Braverman Y, et al. Molecular identification, phylogenetic status, and geographic distribution of Culicoides oxystoma (Diptera: Ceratopogonidae) in Israel. PLoS One 2012;7:e33610.

7. Maan NS, Maan S, Nomikou K, et al. RTPCR assays for seven serotypes of epizootic haemorrhagic disease virus \& their use to type strains from the Mediterranean region and North America. PLoS One 2010;5:e12782.

8. EFSA AHAW Panel. Scientific Opinion on Epizootic Hemorrhagic Disease. EFSA J 2009; 7:1418-85.

9. Temizel EM, Yesilbag K, Batten C, et al. Epizootic hemorrhagic disease in cattle, Western Turkey. Emerg Infect Dis 2009;15:317-9.

10. Anon. National Atlas of Jordan. 1. Climate and Agroclimatology. Amman, Jordan:
Jordan National Geographic Center 1984. $\mathrm{p} 135$.

11. Glukhova VM. Culicoides (Diptera, Ceratopogonidae) of Russia and adjacent lands. Int J Dipterol Res 2005;16:3-75.

12. Cetre-Sossah C, Baldet T, Delecolle JC, et al. Molecular detection of Culicoides spp. and Culicoides imicola, the principal vector of bluetongue (BT) and African horse sickness (AHS) in Africa and Europe. Vet Res 2004;35:325-37.

13. Ortega MD, Holbrook FR, Lloyd JE. Seasonal distribution and relationship to temperature and precipitation of the most abundant species of Culicoides in five provinces of Andalusia, Spain. J Am Mosq Control Assoc 1999;15:391-9.

14. Conte A, Giovannini A, Savini L, et al. The effect of climate on the presence of Culicoides imicola in Italy. J Vet Med B Infect Dis Vet Public Health 2003;50:13947.

15. Baylis M, Bouayoune $\mathrm{H}$, Touti J, et al. Use of climatic data and satellite imagery to model the abundance of Culicoides imicola, the vector of African horse sickness virus, in Morocco. Med Vet Entomol 1998;12:255-66.

16. Mathieu B, Perrin A, Baldet T, et al. Molecular identification of Western European species of obsoletus complex (Diptera: Ceratopogonidae) by an internal transcribed spacer-1 rDNA multiplex polymerase chain reaction assay. $\mathrm{J}$ Med Entomol 2007;44:1019-25.

17. Nolan DV, Carpenter S, Barber J, et al. Rapid diagnostic PCR assays for members of the Culicoides obsoletus and Culicoides pulicaris species complexes, implicated vectors of bluetongue virus in Europe. Vet Microbiol 2007;124:82-94.

18. Pages N, Munoz-Munoz F, Talavera S, et al. Identification of cryptic species of Culicoides (Diptera: Ceratopogonidae) in the subgenus Culicoides and development of species-specific PCR assays based on barcode regions. Vet Parasitol 2009;165:298-310.

19. Wenk CE, Kaufmann C, Schaffner F, et al.
Molecular characterization of Swiss Ceratopogonidae (Diptera) and evaluation of real-time PCR assays for the identification of Culicoides biting midges. Vet Parasitol 2012;184:258-66.

20. Gordon SJ, Bolwell C, Rogers C, et al. The occurrence of Culicoides species, the vectors of arboviruses, at selected trap sites in Zimbabwe. Onderstepoort J Vet Res 2015;82:900.

21. Goffredo M, Savini G, Quaglia M, et al. Orbivirus detection from Culicoides collected on African horse sickness outbreaks in Namibia. Vet Ital 2015;51:17-23.

22. Kheir SM. Seasonal activity of Culicoides bahrainensis Boorman, 1989 (Diptera: Ceratopogonidae) in Saud Arabia. J King Saud U Sci 2010;22:167-72.

23. Morag N, Mullens BA, Gottlieb Y. Assessment of survival and body size variation of Culicoides imicola (Diptera: Ceratopogonidae) as functions of "Candidatus Cardinium" (Bacteroidetes) infection status. Appl Environ Microbiol 2013;79:6260-3.

24. Ruder MG, Stallknecht DE, Howerth EW, et al. Effect of temperature on replication of epizootic hemorrhagic disease viruses in Culicoides sonorensis (Diptera: Ceratopogonidae). J Med Entomol 2015;52:1050-9.

25. Scolamacchia F, VAN DEN Broek J, Meiswinkel R, et al. Principal climatic and edaphic determinants of Culicoides biting midge abundance during the 2007-2008 bluetongue epidemic in the Netherlands, based on OVI light trap data. Med Vet Entomol. 2014;28:143-56.

26. Tarawneh Q, Kadıo lu M. An analysis of precipitation climatology in Jordan. Theor Appl Climatol 2003;74:123-36.

27. Del Rio Lopez R, Miranda MA, ParedesEsquivel C, et al. Recovery rates of bluetongue virus serotypes $1,2,4$ and 8 Spanish strains from orally infected Culicoides imicola in South Africa. Med Vet Entomol 2012;26:162-7. 